University of Nebraska - Lincoln

DigitalCommons@University of Nebraska - Lincoln

Faculty Publications from the Department of Electrical \& Computer Engineering, Department Electrical and Computer Engineering

2012

\title{
Position Extraction from a Discrete Sliding-Mode Observer for Sensorless Control of IPMSMs
}

\author{
Yue Zhao \\ University of Nebraska-Lincoln, yue.zhao@huskers.unl.edu \\ Wei Qiao \\ University of Nebraska-Lincoln, wqiao@engr.unl.edu \\ Long Wu \\ Phoenix International - A John Deere Company, WuLong@JohnDeere.com
}

Follow this and additional works at: https://digitalcommons.unl.edu/electricalengineeringfacpub

Part of the Electrical and Computer Engineering Commons

Zhao, Yue; Qiao, Wei; and Wu, Long, "Position Extraction from a Discrete Sliding-Mode Observer for Sensorless Control of IPMSMs" (2012). Faculty Publications from the Department of Electrical and Computer Engineering. 199.

https://digitalcommons.unl.edu/electricalengineeringfacpub/199

This Article is brought to you for free and open access by the Electrical \& Computer Engineering, Department of at DigitalCommons@University of Nebraska - Lincoln. It has been accepted for inclusion in Faculty Publications from the Department of Electrical and Computer Engineering by an authorized administrator of DigitalCommons@University of Nebraska - Lincoln. 


\section{Position Extraction from a Discrete Sliding-Mode Observer for Sensorless Control of IPMSMs}

\author{
Yue Zhao and Wei Qiao \\ Department of Electrical Engineering \\ University of Nebraska-Lincoln \\ Lincoln, NE 68588-0511 USA \\ yue.zhao@huskers.unl.edu; wqiao@engr.unl.edu
}

\author{
Long $\mathrm{Wu}$ \\ Phoenix International - A John Deere Company \\ 1750 NDSU Research Park Dr. \\ Fargo, ND 58102 USA \\ WuLong@JohnDeere.com
}

\begin{abstract}
Sliding-mode observers (SMOs) offer a promising solution for sensorless control of interior permanent magnet synchronous machines (IPMSMs) due to their excellent robustness to system structure and parameter uncertainty. However, in practical applications, it is challenging for an SMO to achieve a perfect estimation for the back electromagnetic force (EMF) using a finite or relatively lower sampling frequency, especially for high-speed applications. Phase shift, magnitude variation, and heavy noise in the estimated back EMF will cause unexpected errors in rotor position extraction. Thus, advanced rotor position extraction methods are needed to obtain position information from the estimated back EMF. This paper proposes a novel estimated speed feedback algorithm to work together with the conventional inverse tangent method and angle tracking observer (ATO) to extract the rotor position from the extended back EMF obtained from a discrete SMO. The extracted position has reduced oscillations compared to that obtained from traditional methods. The proposed position extraction methods are validated by simulations in MATLAB Simulink as well as experiments on a practical IPMSM drive system.
\end{abstract}

Keywords-Angle tracking observer (ATO); interior permanent magnet synchronous machine (IPMSM); position extraction; sliding-mode observer (SMO); speed feedback

\section{INTRODUCTION}

Interior permanent magnet synchronous machines (IPMSMs) are widely used in electric vehicle systems due to their advantages, such as high efficiency and high power density. Accurate information of rotor position is necessary for highperformance control of IPMSMs. Electromechanical sensors, e.g., resolvers, optical encoders, and hall-effect sensors, are commonly used to obtain rotor position/speed in IPMSM drives. The use of these sensors increases cost, size, weight, and wiring complexity of IPMSM drive systems. From the viewpoint of system reliability, mounting electromechanical sensors on the rotors will reduce the mechanical robustness of electric machines. The electromagnetic interference (EMI) noise and harness break will be fatal to the control system. Moreover, sensors are subjected to high failure rates in harsh environments, such as high environment temperature, highspeed operation, and adverse or heavy load conditions [1]. To overcome these drawbacks, much research effort has gone into the development of sensorless drives that have comparable or similar dynamic performance to the sensor-based drives during the last decades.
The unmodeled dynamics, e.g., nonlinearity and parameter uncertainty, in conventional IPMSM models make the design and implementation of position observers difficult. Among different observers, the sliding-mode observer (SMO) is a promising candidate. If a sliding-mode manifold is well designed, when the state trajectory reaches the manifold, the sliding mode will be enforced [2]-[4]. The system's dynamic behavior under sliding mode only depends on the surfaces chosen in the state space and is not affected by the system structure and parameter uncertainties. SMOs have been applied to surface-mounted PMSM drives in several previous works [6]-[9], where the SMOs used discontinuous functions (e.g., a sign function) to estimate the back EMF based on the errors of the stator current estimation. However, those SMOs used a high sampling frequency (e.g., $20 \mathrm{kHz}$ ) and were not studied for high-speed operations. The IPMSMs drives for electric vehicle applications often need a wide speed range from standstill to 5,000 RPM or even higher. Moreover, considering switching losses, drive size, and EMI issues, the PWM frequency in practical IPMSM drives is normally lower than 10 $\mathrm{kHz}$. Low sampling frequency and high-speed operation make the application of the discrete-time SMO more challenging.

As discussed above, because of unknown parameters, unmodeled dynamics, disturbances, and low sampling frequency, there will be phase shift, magnitude variation, and heavy noise or even distortion in the estimated back EMF. In practical applications, IGBT dead-time effect, inductance and resistance variations, loading condition change, and DC bus variation will affect the performance of discrete-time SMOs. If the conventional inverse tangent method is directly used to extract position information from the estimated back EMF, there will be oscillations in the estimated position, leading to the increase of position errors. In order to solve this problem, a novel estimated speed feedback algorithm is proposed to work together with the conventional inverse tangent method and angle tracking observer (ATO) to mitigate the oscillations in the estimated position.

This paper is organized as follow. Section II gives a brief description on the model of a discrete-time SMO for estimation of the extended back EMF and the rotor position/speed sensorless IPMSM control system. Then, problems incurred in the conventional inverse tangent method are discussed in Section III. The estimated speed feedback algorithm and the 
resulting improved inverse tangent method and ATO are provided in Section IV, followed by experimental results on a practical IPMSM drive. Section VI concludes the paper.

\section{Discrete SMO AND SENSORless CONTROL SySTEM}

\section{A. Mathmatical Model of Discrete-Time SMO}

To facilitate the rotor position observation, an extended back EMF-based model for IPMSMs is proposed in [10] as follows:

$$
\begin{aligned}
& {\left[\begin{array}{c}
v_{\alpha} \\
v_{\beta}
\end{array}\right]=\left[\begin{array}{cc}
R+p L_{d} & \omega_{r e}\left(L_{d}-L_{q}\right) \\
\omega_{r e}\left(L_{q}-L_{d}\right) & R+p L_{d}
\end{array}\right]\left[\begin{array}{l}
i_{\alpha} \\
i_{\beta}
\end{array}\right]} \\
& +\underbrace{\left[\left(L_{d}-L_{q}\right)\left(\omega_{r e} i_{d}-p i_{q}\right)+\omega_{r e} \psi_{m}\right]\left[\begin{array}{c}
-\sin \theta_{r e} \\
\cos \theta_{r e}
\end{array}\right]}_{\text {extended back EMF }}
\end{aligned}
$$

where $p$ is the derivative operator; $v_{\alpha}, v_{\beta}, i_{\alpha}$, and $i_{\beta}$ are the stator voltages and currents in $\alpha-\beta$ stationary reference frame, respectively; $\omega_{r e}$ is the rotor electrical angular speed; $L_{d}$ and $L_{q}$ are the $d$ - and $q$-axis inductances, respectively; and $R$ is the stator resistance.

In (1), only the extended back EMF term contains the information of the rotor position. If the extended back EMF can be estimated, the rotor position can be obtained directly.

Let $\eta$ denote the $\left(L_{d}-L_{q}\right)\left(\omega_{r e} i_{d}-p i_{q}\right)+\omega_{r e} \psi_{m}$ term, the current model of the IPMSM can be written as:

$$
\left\{\begin{array}{l}
\frac{d i_{\alpha}}{d t}=\frac{v_{\alpha}}{L_{d}}-\frac{R}{L_{d}} i_{\alpha}+\omega_{r e} \frac{L_{q}-L_{d}}{L_{d}} i_{\beta}+\frac{\eta}{L_{d}} \sin \theta_{r e} \\
\frac{d i_{\beta}}{d t}=\frac{v_{\beta}}{L_{d}}-\frac{R}{L_{d}} i_{\beta}-\omega_{r e} \frac{L_{q}-L_{d}}{L_{d}} i_{\alpha}-\frac{\eta}{L_{d}} \cos \theta_{r e}
\end{array}\right.
$$

A sliding-mode current observer is designed with the same structure as the IPMSM model:

$$
\left\{\begin{array}{l}
\frac{d \hat{i}_{\alpha}}{d t}=\frac{v_{\alpha}^{*}}{L_{d}}-\frac{R}{L_{d}} \hat{i}_{\alpha}+\omega_{r e} \frac{L_{q}-L_{d}}{L_{d}} i_{\beta}+l \times Z_{\alpha} \\
\frac{d \hat{i}_{\beta}}{d t}=\frac{v_{\beta}^{*}}{L_{d}}-\frac{R}{L_{d}} \hat{i}_{\beta}-\omega_{r e} \frac{L_{q}-L_{d}}{L_{d}} i_{\alpha}-l \times Z_{\beta}
\end{array}\right.
$$

where $l$ is the SMO gain of the switching control vector $Z_{\alpha \beta}$; $v_{\alpha}^{*}$ and $v_{\beta}^{*}$ are commanded voltages obtained from the current regulated vector control of the IPMSM. If the IGBT dead-time effect is well compensated, $v_{\alpha \beta}^{*}$ should be identical to the $v_{\alpha \beta}$ measured from the IPMSM stator terminals. Subtracting (3) from (2) the following equations can be obtained.

$$
\left\{\begin{array}{l}
\frac{d\left(i_{\alpha}-\hat{i}_{\alpha}\right)}{d t}=-\frac{R}{L_{d}}\left(i_{\alpha}-\hat{i}_{\alpha}\right)+\frac{\eta}{L_{d}} \sin \theta_{\mathrm{re}}+l \times Z_{\alpha} \\
\frac{d\left(i_{\beta}-\hat{i}_{\beta}\right)}{d t}=-\frac{R}{L_{d}}\left(i_{\alpha}-\hat{i}_{\alpha}\right)-\frac{\eta}{L_{d}} \cos \theta_{\mathrm{re}}+l \times Z_{\beta}
\end{array}\right.
$$

Let $e_{\alpha \beta}^{T}=\left[\begin{array}{ll}\eta / L_{d} \sin \theta_{r e} & -\eta / L_{d} \cos \theta_{r e}\end{array}\right], Z_{\alpha \beta}^{T}=\left[\begin{array}{ll}Z_{\alpha} & -Z_{\beta}\end{array}\right]$, and $S^{T}=\left[\begin{array}{lll}i_{\alpha}-\hat{i}_{\alpha} & i_{\beta}-\hat{i}_{\beta}\end{array}\right]$, (4) can be expressed as:

$$
\dot{S}^{T}=-\frac{R}{L_{d}} S^{T}+e_{\alpha \beta}^{T}-l \times Z_{\alpha \beta}^{T}
$$

Since the extended back EMF are bounded, they can be suppressed by the discontinuous input with $l>\max \left\{\left|e_{\alpha}\right|,\left|e_{\beta}\right|\right\}$.
When the system is enforced to the sliding mode, $\dot{S}^{T}=S^{T}=0$ and $e_{\alpha \beta}^{T}=l \times Z_{\alpha \beta}^{T}$.

In digital control applications, a discrete-time model of the SMO is needed. To achieve fast calculation and implementation of the switching function in the SMO, the Euler method is used to transform the continuous SMO to a discrete-time observer, which can be expressed as following:

$$
\left\{\begin{array}{l}
\hat{i}_{\alpha}[k+1]=T_{s}\left(\frac{v_{\alpha}^{*}}{L_{d}}+\omega_{r e} \frac{L_{q}-L_{d}}{L_{d}} i_{\beta}[k]+l Z_{\alpha}[k]\right)+\left(1-\frac{T_{s} R}{L_{d}}\right) \hat{i}_{\alpha}[k] \\
\hat{i}_{\beta}[k+1]=T_{s}\left(\frac{v_{\beta}^{*}}{L_{d}}-\omega_{r e} \frac{L_{q}-L_{d}}{L_{d}} i_{\alpha}[k]-l Z_{\beta}[k]\right)+\left(1-\frac{T_{s} R}{L_{d}}\right) \hat{i}_{\beta}[k]
\end{array}\right.
$$

where $T_{s}$ is the sampling period of the SMO, which is normally equal to one PWM cycle in real system applications; $Z_{\alpha}=\operatorname{Sat}\left(i_{\alpha}[k]-\hat{i}_{\alpha}[k]\right)$ and $Z_{\beta}=\operatorname{Sat}\left(i_{\beta}[k]-\hat{i}_{\beta}[k]\right)$, are the outputs of a saturation function, which is used as the switching function in this paper. If the absolute value of the current tracking error is larger than the saturation limit, the output of the saturation function will be the corresponding lower or upper limit. Otherwise, the output of the saturation function will be the current tracking error itself. This linear characteristic of the saturation function will help reduce the step change in the output when compared to the sign function, which will effectively mitigate the output chattering problem of the SMO at steady state.

Since the Euler method is used, the discrete form of current iteration can be expressed as:

$$
i[k+1]=i[k]+T_{s} \frac{\Delta i}{\Delta t}
$$

The current derivative $\Delta i / \Delta t$ can be obtained from (6), and a unit delay of the present step value will be used as previous step value for the next iteration. The block diagram of the current estimator of the discrete SMO is shown in Fig. 1.

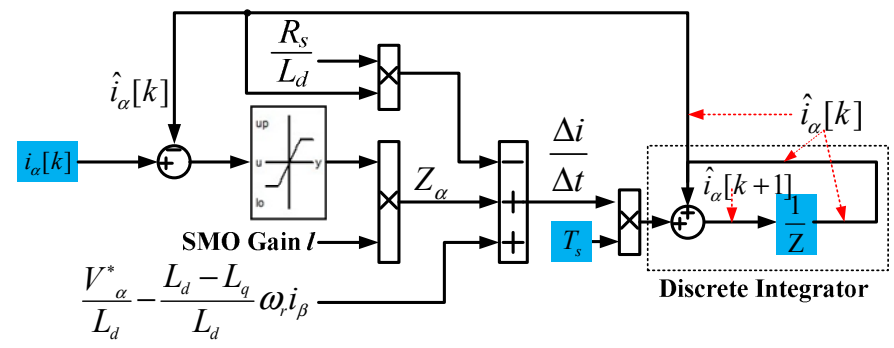

Fig. 1. Block diagram of the $\alpha$-loop current estimator of the discrete SMO.

\section{B. Rotor Position/Speed Sensorless IPMSM Control System}

Fig. 2 shows the overall block diagram of the proposed discrete SMO-based sensorless control system for an IPMSM. The control system consists of a speed PI regulator, which generates a torque command based on speed error. The base torque is the maxium torque at each speed point and is calculated according to the current rotor speed. Since the DCbus voltage also affects the current conmmand, a speed-voltage 


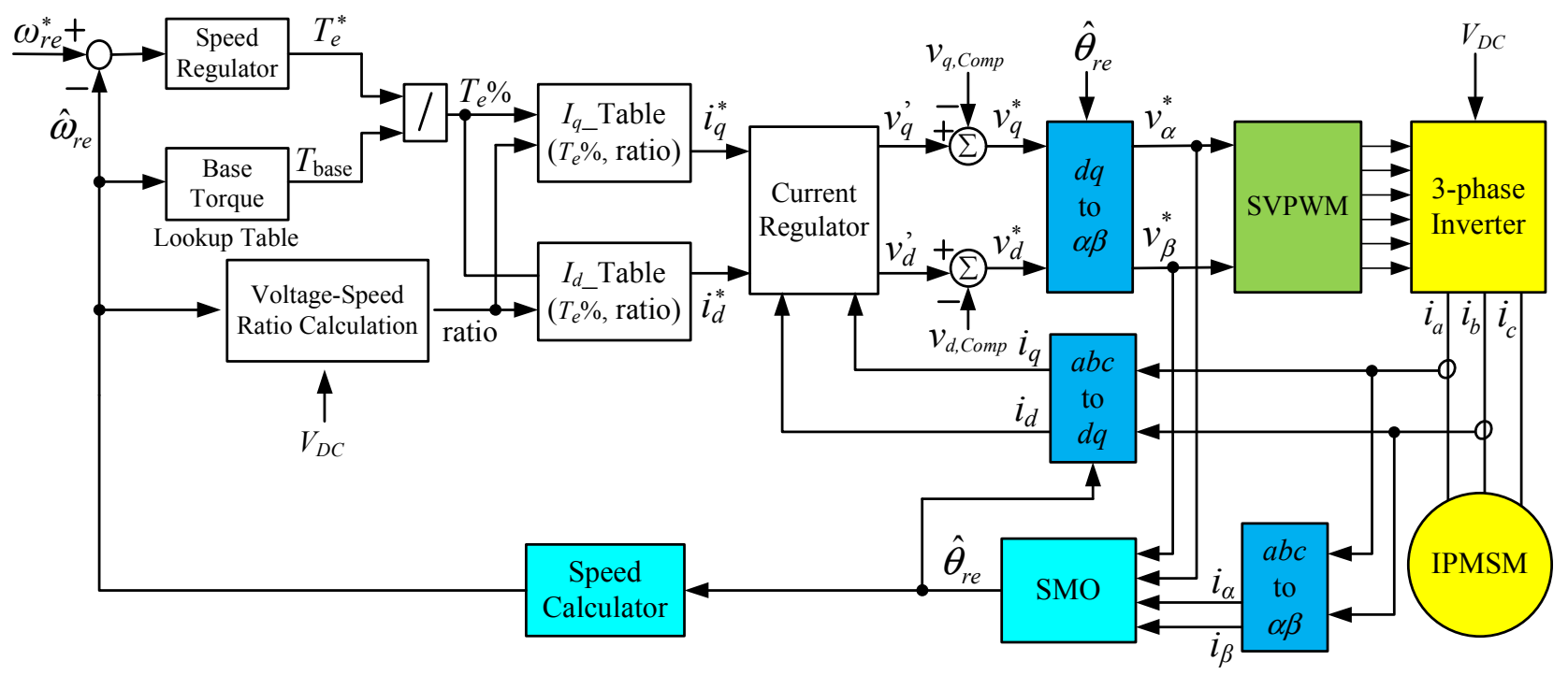

Fig. 2. Block diagram of the proposed sensorless control scheme.

ratio is used. The current commands are calculated based on both torque percentage and speed-voltage ratio. Other modules of the control sytem include current PI regulators with feedforward voltage compensation, Park transformation, spacevector pulse-width modulation (SVPWM), etc.

The rotor position is obtained from the proposed discrete SMO; the rotor speed is then calculated by using a postion buffer based on the estimated rotor position. The buffer size can be ajusted according to the accuracy of the speed and the transient response. The overall system can be tested under both open-loop and closed-loop modes. In the open-loop mode, the SMO uses the commanded voltages $v_{\alpha}^{*}$ and $v_{\beta}^{*}$ and measured currents $i_{a}$ and $i_{b}$ to estimate the rotor position; however the control system still uses the measured rotor position and speed for feedback control. In the closed-loop mode, the estimated rotor position and speed are used by the control system. In the low-speed region, the back EMF is too small to be estimated accurately. Therefore, a starting algorithm is designed to accelerate the IPMSM to a minimum transition speed and then the SMO is enabled.

The major parameters of the IPMSM drive are as follows: the base speed is 5,000 RPM; the stator phase resistance $R_{s}$ is $0.01 \Omega$; the base torque is $400 \mathrm{Nm}$; and the PWM switching frequency is $5 \mathrm{kHz}$. Since the inductances vary with the stator currents, the average values of $L_{d}$ and $L_{q}$ are $0.2 \mathrm{mH}$ and 0.8 $\mathrm{mH}$, respectively. The nominal DC-bus voltage is $700 \mathrm{~V}$. The system is simulated in MATLAB Simulink.

\section{PROBLEM DISCRIPTION}

Per previous discussion, phase shift, magnitude variation, and noise will affect the calculation of rotor position; and these effects are normally present together and bring oscillations to the estimated position, leading to the increase of the position error. In order to understand these effects, simulation studies are carried out and results are shown in Figs. 3 and 4.
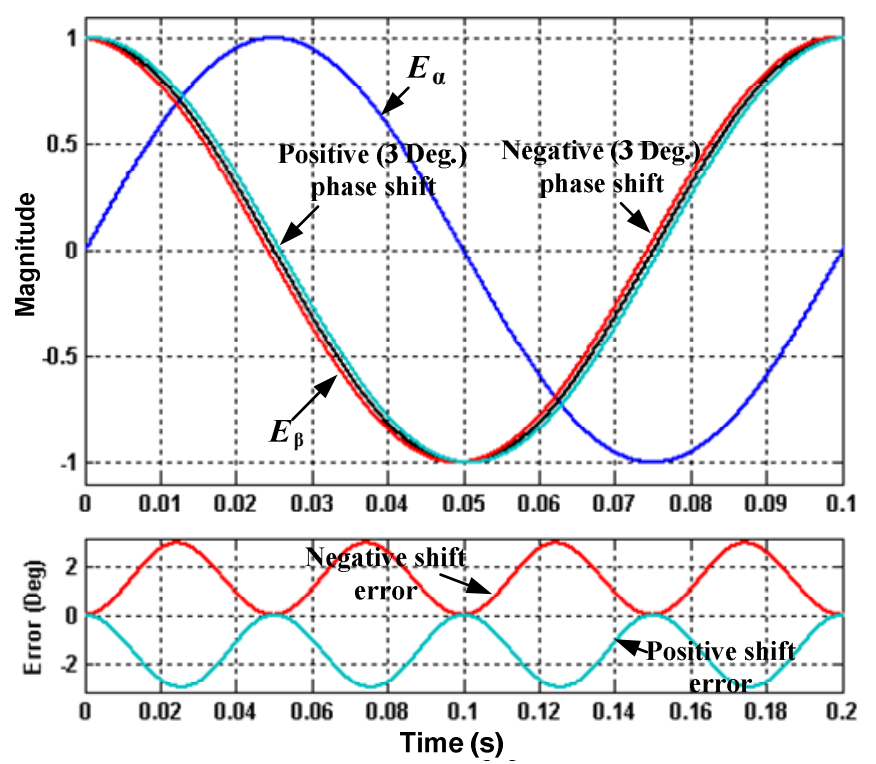

Fig. 3. Effect on the calculated position due to phase shift in the estimated back EMF.

The normalized back EMF (denoted as $E_{\alpha}$ and $E_{\beta}$ ) profiles in $\alpha \beta$ stationary reference frame are shown in Fig. 3. It is assumed that the back EMF profiles have perfect sinusoidal waveform; however, a phase shift has been added intentionally between $E_{\alpha}$ and $E_{\beta}$. In order to evaluate the effect brought by the phase shift between $E_{\alpha}$ and $E_{\beta}$, a negative (leading the original signal) and positive (lagging the original signal) phase shift has been added to $E_{\alpha}$, respectively. As previously mentioned, the inverse tangent method is used to calculate the rotor position based on the back EMF. The errors between the positions calculated from the back EMF with and without the phase shift are also shown in Fig. 3. It is obvious to find that the negative phase shift of 3 electric degrees causes a positive shift in the error waveform from the zero horizon axis. The peak-to-peak 
position error is 3 electric degrees; the average error is 1.5 degrees; and the fundamental frequency of the error waveform is four times the fundamental frequency of the back EMF. The positive phase shift of 3 electric degrees has the similar effect; however, the shift of the error waveform is negative.
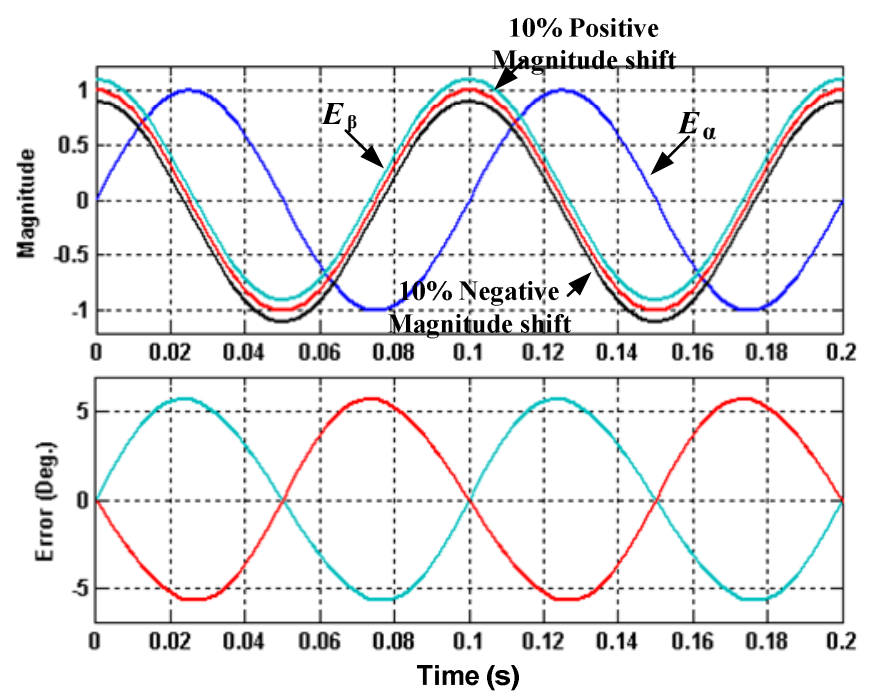

Fig. 4. Effect on the calculated position brought by a magnitude shift in the estimated back EMF.
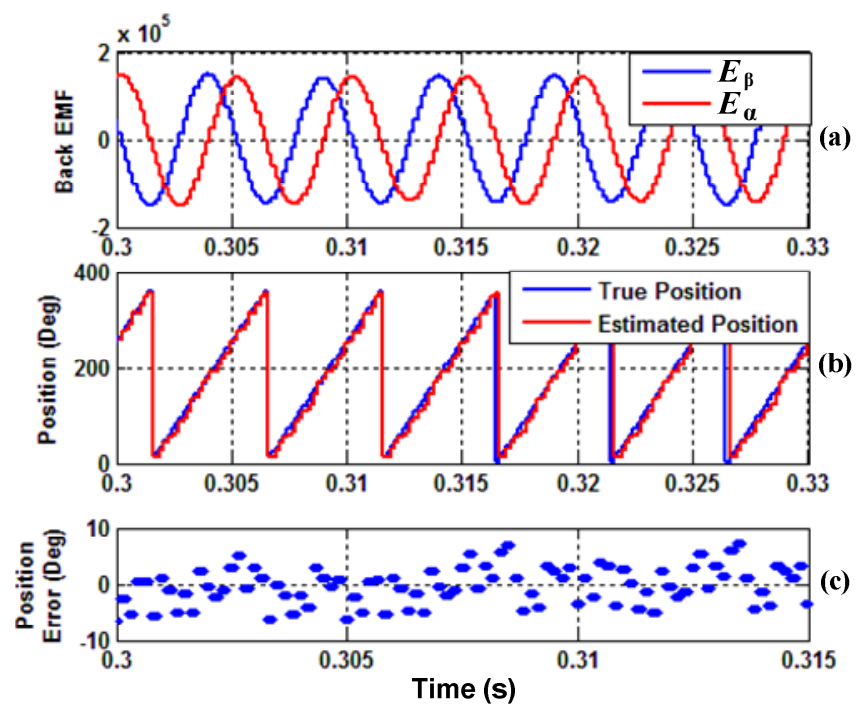

Fig. 5. Simulation results using the directly inverse tangent method. (a) Estimated extended back EMF; (b) measured and estimated positions; and (c) errors between measured and estimated positions.

In order to evaluate the effect on the calculated position brought by a magnitude shift in the estimated back EMF, 10\% negative and positive magnitude shifts have been added to $E_{\alpha}$, respectively, as shown in Fig. 4. The errors between the positions calculated from the back EMF with and without magnitude shift are also shown in Fig. 4. The positive $10 \%$ magnitude shift causes a periodic error waveform symmetric around the zero horizon axis. The magnitude of the position error is 5.7 electric degrees $\left[=\tan ^{-1}(0.1)\right]$; the average error is 0 , and the fundamental frequency of the error waveform is two times the fundamental frequency of the back EMF. The negative $10 \%$ magnitude shift has a similar effect.

The phase and magnitude shifts in the estimated back EMF have different effects on the calculated position. Both the magnitude and fundamental frequency of the position error waveforms are different. The simulation results of the estimated back EMF, estimated and measured positions, and position error are shown in Fig. 5. The effects of phase and magnitude shifts combine together to make the error between the estimated and measured positions to have both phase shift and magnitude oscillation. Because of the switching losses, noise, temperature issue, and CPU loading, a further increase of the sampling frequency cannot be a feasible method to mitigate the phase shift and magnitude oscillation of the position error. Methods that can mitigate these effects need to take into account the physical limitations of the system.

\section{Modified Position EXtRaction Methods}

\section{A. Improved Inverse Tangent Method}

The inverse tangent method is the most straightforward method to extract the rotor position angle from the estimated back EMF. In this method the rotor position angle is determined from the magnitudes of the $\alpha \beta$ back EMF components as follows:

$$
\hat{\theta}=\tan ^{-1}\left(\frac{\hat{e}_{\alpha}}{\hat{e}_{\beta}}\right)
$$

However, the position calculated by this method depends on the quality of the estimated back EMF. Because of the low sampling frequency, the estimated back EMF will have both phase and magnitude shifts, which will bring oscillations and phase shift to the estimated position, as shown in Fig. 5.

In order to mitigate the oscillation of the estimated position, an estimated speed feedback algorithm is proposed to improve the inverse tangent method for position calculation, as shown in Fig. 6. Since the average value of position errors is around zero, as shown in Fig. 5(c), which has also been observed in experimental results, even the estimated rotor position has some oscillations, the rotor speed calculated from the estimated position still has good accuracy, where the errors between the measured and estimated speeds are smaller than $1 \%$. In Fig. 6, $\hat{\theta}_{1}[k]$ is the estimated position obtained from the SMO at the $k^{\text {th }}$ time step; $\widehat{\omega}[k-1]$ is the estimated speed in degree per second at the $(k-1)^{\text {th }}$ time step calculated by using the estimated position. $\hat{\theta}_{2}[k]$, which is a predicted position, can be calculated based on the speed and position at the previous time step.

$$
\hat{\theta}_{2}[k]=\hat{\theta}[k-1]+\omega[k-1] \cdot T_{s}
$$

where $\hat{\theta}[k-1]$ is the position selected form $\hat{\theta}_{1}[k-1]$ and $\hat{\theta}_{2}[k-1]$. The logic for position selection is shown in Fig. 7 .

If the IPMSM drive is enabled, at the $k^{\text {th }}$ time step, $\hat{\theta}_{1}[k]$ can be obtained from the SMO, and $\hat{\theta}_{2}[k]$ has been calculated at the end of the $(k-1)^{\text {th }}$ time step. The error $\varepsilon[k]$ between $\hat{\theta}_{1}[k]$ and $\hat{\theta}_{2}[k]$ will be calculated at the beginning of the $k^{\text {th }}$ time step. If $\varepsilon[k]$ is smaller than the predetermined position error 
margin $\mathrm{E}, \hat{\theta}[k]$ will be equal to $\hat{\theta}_{1}[k]$; otherwise, $\hat{\theta}[k]$ will be equal to $\hat{\theta}_{2}[k]$, which means that the position estimated from the SMO has large oscillations and the magnitude of the oscillation is larger than the error margin. By using this method, the oscillations in the estimated position will be mitigated.

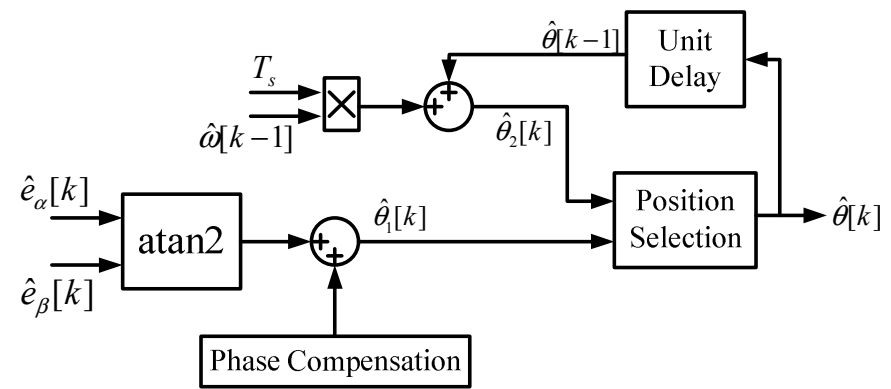

Fig. 6. Block diagram of the improved inverse tangent method.

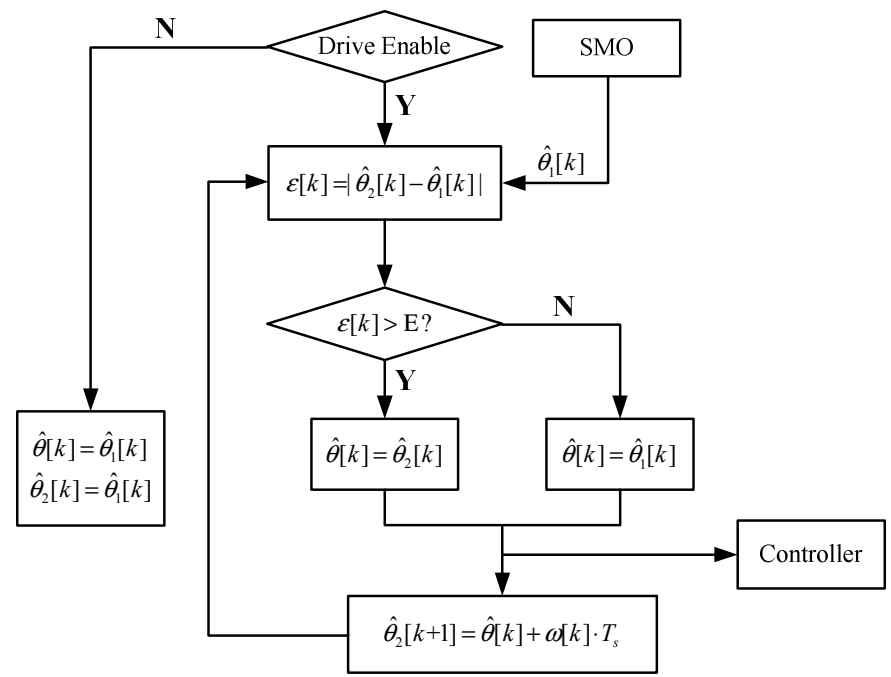

Fig. 7. Logic for position selection.

\section{B. Improved Angle Tracking Observer (ATO)}

The ATO is another widely used angle extraction method, especially in a resolver to digital converter (RDC). Compared to the inverse tangent method, a great advantage of ATO is that it can generate accurate estimation of both rotor position and speed. Another major benefit is its smoothing capability, which is achieved by the combination of an integrator and a proportional-integral (PI) controller. Many methods for selection of the optimal observer coefficients based on transfer functions have been discussed in previous works [11], [12]. If the parameters are properly selected, the ATO will track rotor position and speed precisely. However, because of the distortion in the estimated back EMF, oscillations cannot be eliminated from the estimated position either.

To improve the performance of the ATO, a similar speed feedback algorithm is added to further mitigate the oscillations in the estimated position. The ATO has two outputs: one is the estimated position and the other is the estimated speed. The estimated speed will be used to predict the position $\hat{\theta}_{2}[k]$ in the next time step. The selected position $\hat{\theta}[k]$ is still chosen from $\hat{\theta}_{2}[k]$ and $\hat{\theta}_{1}[k]$, which is the position output of the ATO. The logic for position selection is the same as that shown in Fig. 7.

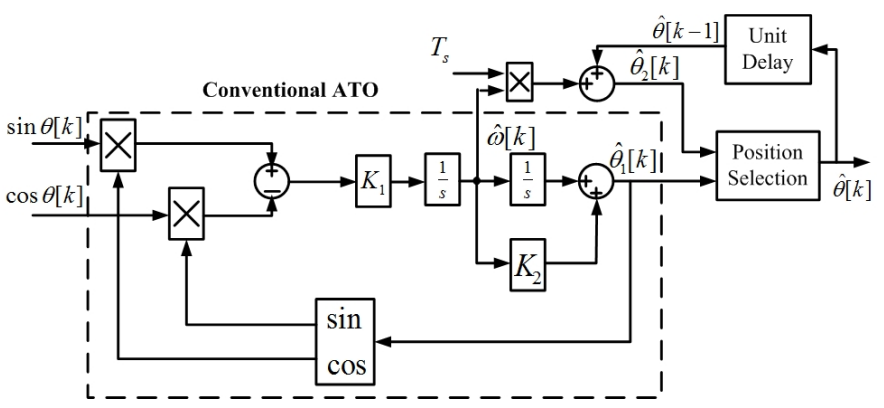

Fig. 8. Block diagram of the improved angle tracking observer (ATO).

\section{EXPERIMENTAL RESULTS}

\section{A. Test Stand Setup}

An experimental stand is designed to verify the proposed algorithms. A brief schematic of the test stand is shown in Fig. 9. In the test stand, a prime mover machine and an IPMSM are connected back to back sharing a common DC bus from a power supply. The prime mover machine maintains the shaft speed while the IPMSM s works in the torque control mode. The base torque is $400 \mathrm{Nm}$ and the base speed is 5,000 RPM for the IPMSM. Considering the issues of current regulation quality, switching losses, system efficiency, switching noise, and EMI, the PWM frequency varies with respect to shaft speed from $2 \mathrm{kHz}$ up to $6 \mathrm{kHz}$. The sampling frequency of the current and voltage is the same with the PWM switching frequency. The SMO is also calculated once per PWM cycle.

\section{B. Experiment Results}

The estimated back EMF by using the conventional inverse tangent method is shown in Fig. 10, when the IPMSM is operated at 4,500 RPM with a sampling frequency of $6 \mathrm{kHz}$. In the estimated back EMF waveforms, both the magnitude and phase shifts can be observed, causing distortions in the back EMF waveforms. Fig. 10 compares the estimated position obtained from the SMO and inverse tangent method and the measured position as well. Most parts of these two positions are on top of each other. However, at some time steps, the difference between them can be easily observed. The simulation results in Fig. 5 are well replicated by the experimental results in Fig. 10.

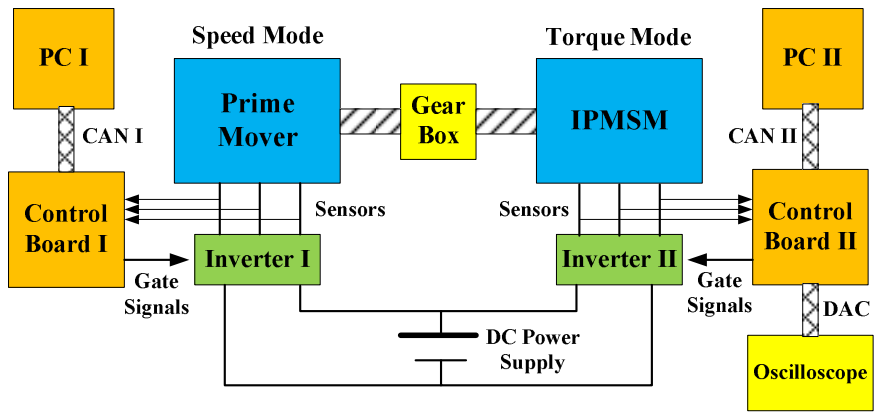

Fig. 9. Schematic of the test stand for an IPMSM. 
In order to mitigate the oscillations in the estimated position, the estimated speed feedback algorithm is added to the inverse tangent method. The experimental results of the estimated and measured positions are compared in Fig. 11, when the IPMSM rotor speed is 2,500 RPM and sampling frequency is $6 \mathrm{kHz}$. The oscillation in the estimated position has been significantly reduced. As shown in the small window on the top of Fig. 11, the estimated and measured rotor positions are on top of each other. In order to see the details clearly, a small positive offset has been added in the measured position. It is obvious to find that the estimated and measured positions are very close to each other in every time step; and the position errors are limited in $+/-3$ electric degrees at steady state.

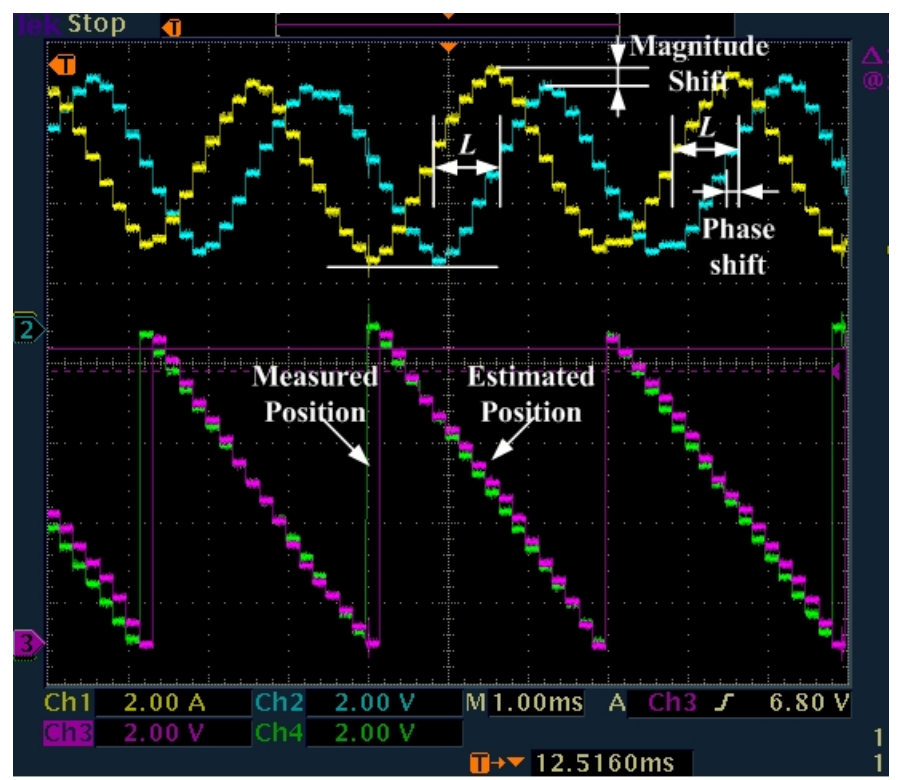

Fig. 10. Experimental results of the extended back EMF, measured and estimated positions using directly the inverse tangent method.

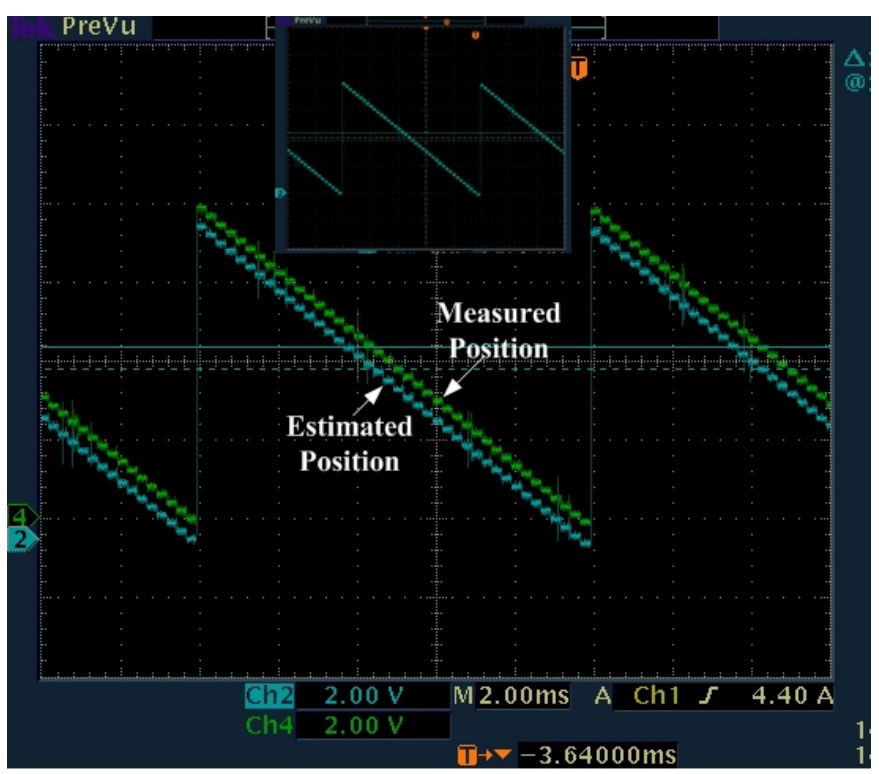

Fig. 11. Experimental results of the measured and estimated positions using the improved inverse tangent method.

\section{CONCLUSION}

A discrete sliding-mode position observer has been proposed for sensorless control of IPMSMs in medium and high-speed range. Due to the limitation in sampling frequency and computational source, it is challenging to achieve a perfect estimation for the extended back EMF. This has caused oscillations in the estimated rotor position using the conventional inverse tangent method or ATO. A speed feedback algorithm has been proposed to improve the inverse tangent method and ATO for rotor position extraction. Experiment results have shown great improvement in the estimated position by using the proposed methods. The errors between the estimated and measured positions have been limited within $+/-3$ electric degrees at steady state with relatively low sampling frequencies up to $6 \mathrm{kHz}$.

\section{ACKNOWLEDGEMENT}

The authors gratefully acknowledge financial support for this work from Phoenix International-A John Deere Company and the U.S. National Science Foundation under grant ECCS0901218 .

\section{REFERENCES}

[1] M. Pacas, "Sensorless drives in industrial applications," IEEE Industrial Electronics Magazine, vol. 5, no. 2, pp. 16-23, June 2011.

[2] R. DeCarlo, S. Zak, and G. Mathews, "Variable structure control of nonlinear multivariable systems: A tutorial," Proc. IEEE, vol. 76, pp. 212-232, Mar. 1988.

[3] V. Utkin, "Variable structure systems with sliding modes," IEEE Trans. Automatic Control, vol. 22, no. 2, pp. 212-222, Apr. 1977.

[4] V. Utkin, J. Guldner, and J. Shi, Sliding Mode Control in Electromechanical Systems, 1st ed. New York: Taylor \& Francis, 1999.

[5] S. Chi, Z. Zhang, and L. Xu, "Sliding-mode sensorless control of directdrive PM synchronous motors for washing machine Applications," IEEE Trans. Industry Applications, vol. 45, no. 2, pp. 582-590, Mar-Apr 2009.

[6] Y. Zhang and V. Utkin, "Sliding mode observers for electric machinesan overview," in Proc. $28^{\text {th }}$ Annual Conference of the IEEE Industrial Electronics Society, Nov. 5-8, 2002, pp. 1842-1847.

[7] S. Chi and L. Xu, "Position sensorless control of PMSM based on a novel sliding mode observer over wide speed range," in Proc. 2006 Power Electronics and Motion Control Conference, vol. 3, pp. 1-7.

[8] M. Elbuluk and C. Li, "Sliding mode observer for wide-speed sensorless control of PMSM drives," in Proc. 2003 IEEE IAS Annual Meeting, vol. 1, pp. 480- 485, 2003.

[9] G. Foo and M. F. Rahman, "Sensorless sliding-mode MTPA control of an IPM synchronous motor drive using a sliding-mode observer and HF signal injection," IEEE Trans. Industrial Electronics, vol. 57, no. 4, pp. 1270-1278, Apr 2010.

[10] Z. Chen, M. Tomita, S. Doki, and S. Okuma, "An extended electromotive force model for sensorless control of interior permanentmagnet synchronous motors," IEEE Trans. Industrial Electronics, vol. 50, no. 2, pp. 288- 295, Apr 2003.

[11] Y. Zhao, W. Qiao, and L. Wu, "Compensation algorithms for sliding mode observers in sensorless control of IPMSMs," in Proc. 2012 IEEE International Electrical Vehicle Conference, Greenville, SC, USA.

[12] R. Hoseinnezhad, and P. Harding, "A novel hybrid angle tracking observer for resolver to digital conversion," in Proc. 44th IEEE Conference on Decision and Control and 2005 European Control Conference, Dec. 12-15, 2005, pp. 7020- 7025.

[13] K. Bouallaga, L. Idkhajine, A.Prata, and E. Monmasson, "Demodulation methods on fully FPGA-based system for resolver signals treatment," in Proc. 2007 European Conference on Power Electronics and Applications, Sept. 2-5, 2007. 